Annals of Pure and Applied Mathematics

Vol. 13, No. 2, 2017, 211-222

ISSN: 2279-087X (P), 2279-0888(online)

Published on 14 April 2017

www.researchmathsci.org

DOI: http://dx.doi.org/10.22457/apam.v13n2a7

Annals of

Pure and Applied

Mathematics

\title{
Interval-valued Q-Fuzzy Ideals Generated by an Interval-valued Q-Fuzzy Subset in Ordered Semi-groups
}

\author{
P. Murugadas ${ }^{1}$, A. Arikrishnan ${ }^{2}$ and M.R. Thirumagal ${ }^{3}$ \\ Department of Mathematics, Annamalai University \\ Annamalainagar-608002, Tamil Nadu, India \\ ${ }^{1}$ Corresponding author. E-mail: bodi_muruga@ yahoo.com; \\ E-mail: ${ }^{2}$ malarsan333@yahoo.com; ${ }^{3}$ r.thirumagal@gmail.com
}

Received 28 February 2017; accepted 14 March 2017

Abstract. This paper expose a study on interval-valued Q-fuzzy ideals generated by an interval-valued Q-fuzzy subset on ordered semi-groups. Some characterizations of such generated interval-valued Q-fuzzy ideals are also discussed.

Keywords: Ordered semi-group, interval-valued Q-fuzzy sub semi-group, interval-valued Q-fuzzy left(right, two-sided) ideal, interval-valued Q-fuzzy interior ideal, interval-valued Q-fuzzy bi-ideal.

AMS Mathematics Subject Classification (2010): 03E72, 08A72,13A15

\section{Introduction}

The important concept of a fuzzy set introduced by Zadeh in 1965 (see [18]) has opened up keen insights and applications in wide range of scientific fields. Rosenfeld introduced the concept of fuzzy groups [15]. Among others, fuzzy semi-groups were introduced by Kuroki [10]. A theory of fuzzy sets on ordered semi-groups has been recently developed $[4,5,6,8,9]$. Fuzzy sets in ordered semi-groups were first studied by Kehayopulu and Tsingelis in [5], then they defined fuzzy analogies for several notations, which have proved useful in the theory of ordered semi-group. In [7], they have discussed fuzzy bi-ideals in ordered semi-groups and they discuss fuzzy interior ideals in ordered semi-group in [9]. Fuzzy semi-groups were generalized in two folds: fuzzy ordered semi-groups and fuzzy ternary semi-groups. Since ordered semi-groups are useful for computer science, especially in theory of automata and formal language, fuzzy ordered semi-group has been extensively studied (see $[1,2,3,5,6])$. Interval-valued fuzzy subsets were proposed thirty years ago as a natural extension of fuzzy sets by Zadeh [18]. In [18], Zadeh also constructed a method of approximate inference using his interval-valued fuzzy subsets. In [13], Narayanan and Manikantan introduced the notions of interval-valued fuzzy ideals generated by an interval-valued fuzzy subset in semi-groups. Shabir and Khan [16] have studied about interval-valued fuzzy ideals generated by an interval-valued fuzzy subset in ordered semi-groups. Thillaigovindan and Chinnadurai [17] initiated some study on Interval-valued fuzzy generalized bi-ideals. Some interesting studies are carried out in 
P.Murugadas, A.Arikrishnan and M.R.Thirumagal

$[11,12,14]$ using interval-valued fuzzy set. This paper characterize the ordered semi-groups in terms of interval-valued Q-fuzzy left (right, interior and bi-)ideals.

\section{Preliminaries}

In [8], Kehayopulu and Tsingelis used the operation $\wedge$ on the unit interval $[0,1]$ to define an operation 'o' on $\mathcal{F}(S)$ as follows:

$$
(f \circ g)(x)=\left\{\begin{array}{cc}
\mathrm{V}_{y, z \in S_{x}} f(y) \wedge g(z) \text { if } S_{x} \neq \emptyset, \text { for all } x \in S, \\
0 \quad \text { otherwise, }
\end{array}\right.
$$

where $S_{x}=\{(y, z) \in S \times S: x \leq y z\}$

An ordered semi-group is an ordered set $S$ at the same time a semi-group such that a $\leq b \Rightarrow a x \leq b x$ and $x a \leq x b$ for all $\forall a, b, x \in S$. .

A non-empty subset $A$ of an ordered semi-group $S$ is called a sub semi-group of $S$ if $A A \subseteq A$.

A non-empty subset $A$ of an ordered semi-group $S$ is called a right (resp. left) ideal of $S$ if

1. $A S \subseteq A$ (resp. $S A \subseteq A$ )

2. $a \in A, S$ э $b \leq a \Rightarrow b \in A$.

$A$ is called an ideal of $S$ if it is both a right and a left ideal of $S$.

A sub semi-group $A$ of an ordered semi-group $S$ is called an interior ideal of $S$ if

1. $S A S \subseteq A$.

2. $a \in A, S$ э $b \leq a \Rightarrow b \in A$.

A semi-group $A$ of an ordered semi-group $S$ is called a bi-ideal of $S$ if

1. $A S A \subseteq A$.

2. $a \in A, S$ э $b \leq a \Rightarrow b \in A$.

A fuzzy subset $f$ of an ordered semi-group $S$ is a function from $S$ to the unit interval [0,1] [5]. Let $S$ be an ordered semi-group. A fuzzy subset $f$ of $\mathrm{S}$ is called a fuzzy sub semi-group of $S$ if $f(x y) \geq \min \{f(x), f(y)\} \forall x, y \in S$.

A fuzzy subset $f$ of an ordered semi-group $S$ is called a fuzzy left (resp. right) ideal of $S$ if

1) $x \leq y \Rightarrow f(x) \geq f(y)$

2) $f(x y) \geq f(y) \quad$ (resp. $f(x y) \geq f(x))$.

If $f$ is both a fuzzy left ideal and a fuzzy right ideal of $S$, then $f$ is called a fuzzy ideal of $S$ or a fuzzy two sided ideal of $S$. Equivalently, $f$ is called a fuzzy ideal of $S$ if

1) $x \leq y \Rightarrow f(x) \geq f(y)$

2) $f(x y) \geq \sup \{f(x), f(y)\}$.

A fuzzy sub semi-group $f$ of an ordered semi-group $S$ is called a fuzzy interior ideal of $S$ if 
IV Q-Fuzzy Ideals Generated by an IV Q-Fuzzy Subset in Ordered Semi-groups

1) $x \leq y \Rightarrow f(x) \geq f(y)$

2) $f(x y z) \geq f(y)$.

A fuzzy sub semi-group $f$ of an ordered semi-group $S$ is called a fuzzy bi-ideal of $S$ if

1) $x \leq y \Rightarrow f(x) \geq f(y)$

2) $f(x y z) \geq \min \{f(x), f(y)\}$.

\section{Interval-valued Q-fuzzy ideals generated by an interval-valued Q-fuzzy subset in ordered semi-groups \\ We now give the interval-valued Q-fuzzy concepts.}

An interval number on [0,1], say $\bar{a}$ is a closed sub-interval of [0,1], that is $\bar{a}=\left[a^{-}, a^{+}\right]$where $0 \leq a^{-} \leq a^{+} \leq 1$. Let $\mathrm{D}[0,1]$ denote the family of all closed sub-intervals of $[0,1], \overline{0}=[0,0]$ and $\overline{1}=[1,1]$. For any two elements $\bar{a}=\left[a^{-}, a^{+}\right]$and $\bar{b}=\left[b^{-}, b^{+}\right]$in $\mathrm{D}[0,1]$. We define
(i) $\bar{a} \leq \bar{b}$ if and only if $a^{-} \leq b^{-}$and $a^{+} \leq b^{+}$
(ii) $\bar{a}=\bar{b}$ if and only if $a^{-}=b^{-}$and $a^{+}=b^{+}$,
(iii) $\operatorname{Min}^{i}\{\bar{a}, \bar{b}\}=\left[\min \left\{a^{-}, b^{-}\right\}, \min \left\{a^{+}, b^{+}\right\}\right]$.
(iv) $\operatorname{Max}^{i}\{\bar{a}, \bar{b}\}=\left[\max \left\{a^{-}, b^{-}\right\}, \max \left\{a^{+}, b^{+}\right\}\right]$.

Similarly we can define $\inf ^{i}$ and $\sup ^{i}$ in case of family of elements in $D[0,1]$.

A mapping $\bar{A}: X \times Q \rightarrow D[0,1]$ is called an interval-valued Q-fuzzy subset of $X$.

where $\bar{A}(x, q)=\left[A^{-}(x, q), A^{+}(x, q)\right] . \forall x \in X$ and $q \in Q$.

Let $S$ be an ordered semi-group with identity element 1 and IQF(S) denotes the set of all i-v Q-fuzzy subsets of $S$.

Definition 3.1. Let $\bar{A}, \bar{B}$ be $i$-v $Q$-fuzzy subsets of $S$. Then we have the following:

(i) $\bar{A} \leq \bar{B}$ if and only if $\bar{A}(x, q) \leq \bar{B}(x, q)$.

(ii) $\bar{A}=\bar{B}$ if and only if $\bar{A}(x, q)=\bar{B}(x, q)$.

(iii) $(\bar{A} \cup \bar{B})(x, q)=\max ^{i}\{\bar{A}(x, q), \bar{B}(x, q)\}$.

(iv) $(\bar{A} \cap \bar{B})(x, q)=\min ^{i}\{\bar{A}(x, q), \bar{B}(x, q)\}$, for all $x \in S$ and $q \in Q$.

Definition 3.2. Let "०" be a binary composition in $S$. The product $\bar{A} \circ \bar{B}$ of any two $i-v$ $Q$-fuzzy subsets $\bar{A}, \bar{B}$ of $S$ is defined by

$$
(\bar{A} \circ \bar{B})(\mathrm{x}, \mathrm{q})=\left\{\begin{array}{cc}
\mathrm{V}_{x=a b}^{i}\left\{\operatorname{Min}^{i}\{\bar{A}(a, q), \bar{B}(b, q)\}\right\} & \text { if } x \text { is expressible as } x=a b \\
\overline{0} & \text { otherwise }
\end{array}\right.
$$

Since semi-group $S$ is associative, The operation "o" is associative.

We denote $x y$ instead of $x \cdot y$ and $\bar{A} \bar{B}$ for $\bar{A} \circ \bar{B}$. Let $B$ be a subset of a set $S$. 
P.Murugadas, A.Arikrishnan and M.R.Thirumagal

Define a function (characteristic function) $\bar{\chi}_{B}: S \times Q \rightarrow \mathrm{D}[0,1]$ by

$$
\bar{\chi}_{B}(x, q)=\left\{\begin{array}{cc}
\overline{1} \text { if } x \in B & \forall x \in S \text { and } q \in Q . \\
\overline{0} & \text { otherwise }
\end{array}\right.
$$

clearly $\bar{\chi}_{B}$ is an $i-v$ Q-fuzzy subset of $S$. Throughout this paper $\bar{\chi}_{s}$ is denoted by $\bar{S}$ and $\mathrm{S}$ will denote a semi-group unless mentioned.

Definition 3.3. Let $A$ be a $Q$-fuzzy subset of an ordered semi-group $S$ with identity element 1. Then the smallest Q-fuzzy left (right, two sided, interior, bi-)ideal of $S$ containing $A$ is called a $Q$-fuzzy left (right, twosided,interior, bi-)ideal of $S$ generated by $A$ denoted by $\langle A\rangle_{L}\left(\langle A\rangle_{R},\langle A\rangle,\langle A\rangle_{I},\langle A\rangle_{B}\right)$ respectively.

Definition 3.4. An i-v $Q$-fuzzy subset $\bar{A}$ of an ordered semi-group $S$ is called an $i-v$ $Q$-fuzzy sub semi-group of $S$ if for all $x, y \in S$ and $q \in Q$,

$$
\bar{A}(x y, q) \geq \operatorname{Min}^{i}\{\bar{A}(x, q), \bar{A}(y, q)\} .
$$

Definition 3.5. An $i$-v $Q$-fuzzy subset $\bar{A}$ of an ordered semi-group $S$ is called an $i-v$ $Q$-fuzzy left (resp. right) ideal of $S$ if for all $x, y \in S$ and $q \in Q$

i) $x \leq y \Rightarrow \bar{A}(x, q) \geq \bar{A}(y, q)$ and $q \in Q$

ii) $\bar{A}(x y, q) \geq \bar{A}(y, q)$ (resp. $\bar{A}(x y, q) \geq \bar{A}(x, q))$.

An i-v Q-fuzzy subset $\bar{A}$ in $S$ is called an interval valued fuzzy two sided ideal of $S$ if it is an i-v Q-fuzzy left ideal and an i-v Q-fuzzy right ideal of $S$.

Definition 3.6. An $i$-v $Q$-fuzzy sub semi-group $\bar{A}$ of an ordered semi-group $S$ is called an $i$-v $Q$-fuzzy interior ideal of $S$ if for all $x, y, z \in S$ and $q \in Q$
i) $x \leq y \Rightarrow \bar{A}(x, q) \geq \bar{A}(y, q)$
ii) $\bar{A}(x y z, q) \geq \bar{y}$.

Definition 3.7. An i-v $Q$-fuzzy sub semi-group $\bar{A}$ of an ordered semi-group $S$ is called an $i$-v $Q$-fuzzy bi-ideal of $S$ iffor all $x, y, z \in S$ and $q \in Q$

i) $x \leq y \Rightarrow \bar{A}(x, q) \geq \bar{A}(y, q)$

ii) $\bar{A}(x y z, q) \geq \operatorname{Min}^{i}\{\bar{A}(x, q), \bar{A}(z, q)\}$.

Theorem 3.8. An interval-valued $Q$-fuzzy subset $\bar{A}$ of an ordered semi-group $S$ is an $i$-v $Q$-fuzzy sub semi-group (left ideal, right ideal, two sided ideal, interior ideal, bi-ideal) of $S$ iff $A^{-}$and $A^{+}$are $Q$-fuzzy sub semi-group (left ideals, right ideals, interior ideals, bi-ideals) of $S$.

Proof: Let $\bar{A}$ be an i-v Q-fuzzy sub semi-group of $S$. 
IV Q-Fuzzy Ideals Generated by an IV Q-Fuzzy Subset in Ordered Semi-groups

Then for all $x, y \in S$, and $q \in Q \bar{A}(x y, q) \geq \operatorname{Min}^{i}\{\bar{A}(x, q), \bar{A}(y, q)\}$, where $\operatorname{Min}^{i}\{\bar{A}(x, q), \bar{A}(y, q)\}=\left[\min \left\{A^{-}(x, q), A^{-}(y, q)\right\}, \min \left\{A^{+}(x, q), A^{+}(y, q)\right\}\right]$.

Thus $\bar{A}(x y, q) \geq\left[\min \left\{A^{-}(x, q), A^{-}(y, q)\right\}, \min \left\{A^{+}(x, q), A^{+}(y, q)\right\}\right]$.

Hence $\left[A^{-}(x y, q), A^{+}(x y, q) \geq\left[\min \left\{A^{-}(x, q), A^{-}(y, q)\right\}, \min \left\{A^{+}(x, q), A^{+}(y, q)\right\}\right]\right.$.

Thus $A^{-}(x y, q) \geq \min \left\{A^{-}(x, q), A^{-}(y, q)\right\}$ and $\left.A^{+}(x y, q) \geq \min \left\{A^{+}(x, q), A^{+}(y, q)\right\}\right]$.

Hence $A^{-}, A^{+}$are Q-fuzzy sub semi-groups of $S$. The converse is straightforward.

Now suppose $\bar{A}$ is an i-v Q-fuzzy left ideal of $S$.

Then for all $x, y \in S$, and $q \in Q \quad x \leq y \Rightarrow \bar{A}(x, q) \geq \bar{A}(y, q)$ and $\bar{A}(x y, q) \geq \bar{A}(y, q)$

Now $\bar{A}(x, q) \geq \bar{A}(y, q) \Rightarrow\left[A^{-}(x, q), A^{+}(x, q) \geq\left[A^{-}(y, q), A^{+}(y, q)\right]\right.$.

That is $A^{-}(x, q) \geq A^{-}(y, q)$ and $A^{+}(x, q) \geq A^{+}(y, q)$.

$\bar{A}(x y, q) \geq \bar{A}(y, q) \Rightarrow\left[A^{-}(x y, q)\right], A^{+}(x y, q) \geq\left[A^{-}(y, q), A^{+}(y, q)\right]$.

Thus $A^{-}(x y, q) \geq A^{-}(y, q)$ and $A^{+}(x y, q) \geq A^{+}(y, q)$.

Hence, $A^{-}$and $A^{+}$are Q-fuzzy left ideals of $S$.

The converse is straightforward. Similarly we can prove for other cases.

Definition 3.9. Let $\bar{A}$ be an $i$-v $Q$-fuzzy set. Then the smallest $i$-v $Q$-fuzzy left (right, two sided) ideal of $S$ containing $\bar{A}$ is called an $i$-v $Q$-fuzzy left ( right, two sided) ideal of $S$ generated by $\bar{A}$, denoted by $\langle\bar{A}\rangle_{L}\left(\left\langle\bar{A}_{R}\right\rangle,\langle\bar{A}\rangle\right)$ respectively.

Theorem 3.10. Let $\bar{A}$ be an $i-v$-fuzzy set. Then $\langle\bar{A}\rangle_{L}=\bar{J}$,

where $\bar{J}=\left[J^{-}, J^{+}\right]$such that

$J^{-}(x, q)=\sup _{\substack{x \leq x_{1} x_{2} \\ x_{1}, x_{2} \in S}} A^{-}\left(x_{2}, q\right)$ and $J^{+}(x, q)=\sup _{\substack{x \leq x_{1} x_{2} \\ x_{1}, x_{2} \in S}} A^{+}\left(x_{2}, q\right)$ for all $x \in S$ and $q \in Q$.

Proof: For all $a \in S$ and $q \in Q$,

$J^{-}(a, q)=\sup _{a \leq x_{1} x_{2}} A^{-}\left(x_{2}, q\right) \geq A^{-}(a, q)$, since $a=1 . a \Rightarrow A^{-}(a, q) \leq J^{-}(a, q)$.

Similarly $A^{+}(a, q) \leq J^{+}(a, q) \Rightarrow \bar{A}(a, q)=\left[A^{-}(a, q), A^{+}(a, q)\right] \leq\left[J^{-}(a, q), J^{+}(a, q)\right]$.

Thus $\bar{A} \subseteq \bar{J}$. Now we show that $\bar{J}$ is an interval-valued Q-fuzzy left ideal of $S$, for this we have to show $(x, q) \leq(y, q) \Rightarrow \bar{J}(x, q) \geq \bar{J}(y, q)$ and $\bar{J}(x y, q) \geq \bar{J}(y, q)$ for all $x, y \in S$ and $q \in Q$. Let $x, y \in S$, such that $x \leq y$. If $y \leq x_{3} x_{4}$, then $x \leq x_{3} x_{4}$.

Hence $J^{-}(y, q)=\sup _{y \leq x_{3} x_{4}} A^{-}\left(x_{4}, q\right) \leq \sup _{x \leq x_{1} x_{2}} A^{-}\left(x_{2}, q\right)=J^{-}(x, q)$.

Similarly $J^{+}(x, q) \geq J^{+}(y, q) \Rightarrow \bar{J}(x, q) \geq \bar{J}(y, q)$.

Now if $y \leq x_{1} x_{2}$, then $(x y, q) \leq\left(\left(x x_{1}\right) x_{2}, q\right)$. 
P.Murugadas, A.Arikrishnan and M.R.Thirumagal

Hence $J^{-}(y, q)=\sup _{y \leq x_{1} x_{2}} A^{-}\left(x_{2}, q\right) \leq \sup _{x y \leq x_{3} x_{4}} A^{-}\left(x_{4}, q\right)=J^{-}(x y, q)$.

Similarly $J^{+}(x y, q) \geq J^{+}(y, q)$. Hence $\bar{J}(x y, q) \geq \bar{J}(y, q)$.

Let $\bar{B}$ be any i-v Q-fuzzy left ideal of $S$ such that $\bar{B} \supseteq \bar{A}$.

Then for all $a \in S$ and $q \in Q, B^{-}(a, q) \geq A^{-}(a, q)$ and $B^{+}(a, q) \geq A^{+}(a, q)$. Now

$J^{-}(a, q)=\sup _{a \leq a_{1} a_{2}} A^{-}\left(a_{2}, q\right) \leq \sup _{a \leq a_{1} a_{2}} B^{-}\left(a_{2}, q\right) \leq \sup _{a \leq a_{1} a_{2}} B^{-}\left(a_{1} a_{2}, q\right) \leq B^{-}(a, q)$.

Similarly, $B^{+}(a, q) \geq J^{+}(a, q)$ for all $a \in S$ and $q \in Q \Rightarrow \bar{J} \subseteq \bar{B}$.

Hence, $\langle\bar{A}\rangle_{L}=\bar{J}$.

Theorem 3.11. Let $\bar{A}$ be an $i-v Q$-fuzzy set, Then $\langle\bar{A}\rangle_{R}=\bar{J}$,

where $\bar{J}=\left[J^{-}, J^{+}\right]$such that

$J^{-}(x, q)=\sup _{\substack{x \leq x_{1} x_{2} \\ x_{1}, x_{2} \in S}} A^{-}\left(x_{1}, q\right)$ and $J^{+}(x, q)=\sup _{\substack{x \leq x_{1} x_{2} \\ x_{1}, x_{2} \in S}} A^{+}\left(x_{1}, q\right)$ for all $x \in S$.

Proof: The proof is similar to the proof of Theorem 3.10.

Theorem 3.12. Let $\bar{A}$ be an $i$-v $Q$-fuzzy set. An interval-valued $Q$-fuzzy subset $\bar{J}$ is an $i$-v $Q$-fuzzy left (right) ideal of $S$ generated by $\bar{A}$ if and only if $J^{-}$and $J^{+}$are $Q-f u z z y$ left (right) ideals of $S$ generated by $A^{-}$and $A^{+}$respectively.

Proof: Suppose $\bar{J}$ is an i-v Q-fuzzy left ideal of $S$ generated by $\bar{A}$.

Then by Theorem 3.8, $J^{+}$and $J^{-}$are Q-fuzzy left ideals of $S$.

Since $\bar{A} \subseteq \bar{J}$, we have $A^{-} \subseteq J^{-}$and $A^{+} \subseteq J^{+}$. If $B$ is a Q-fuzzy left ideal of $\mathrm{S}$ containing $A^{-}$, then define $\bar{B}: S \times Q \rightarrow \mathrm{D}[0,1]$ by $\bar{B}(x, q)=\left[B^{-}(x, q), B^{-}(x, q)\right]$, where $B^{-}(x, q)=B(x, q)$ for all $x \in S$ and $B^{+}(x, q)=1$ for all $x \in S$ and $q \in Q$.

Since $B^{-}$and $B^{+}$are Q-fuzzy left ideals of $S$, by Theorem 3.8, $\bar{B}$ is an i-v Q-fuzzy left ideal of $S$. Clearly $\bar{A} \subseteq \bar{B}$ so $\bar{J} \subseteq \bar{B} \Rightarrow J^{-} \subseteq B^{-}=B \Rightarrow J^{-}$is Q-fuzzy left ideal of $S$ generated by $A^{-}$. Similarly we can show that $J^{+}$is Q-fuzzy left ideal of $S$ generated by $A^{+}$.

Conversely, assume that $J^{-}$and $J^{+}$are Q-fuzzy left ideals of S generated by $A^{-}$and $A^{+}$respectively.Then by Theorem 3.8, $\bar{J}$ is an i-v Q-fuzzy left ideals of $S$ containing $\bar{A}$. If $\bar{B}$ is an i-v Q-fuzzy left ideal of $S$ containing $\bar{A}$, then $A^{-} \subseteq B^{-}$and $A^{+} \subseteq B^{+}$ Since $B^{-}$and $B^{+}$are Q-fuzzy left ideals of $S$, we have $J^{-} \subseteq B^{-}$and $J^{+} \subseteq B^{+}$. Hence $\bar{J} \subseteq \bar{B}$. Thus $\bar{J}$ is an i-v Q-fuzzy left ideal of $S$ generated by $\bar{A}$.

Theorem 3.13. Let $\bar{A}$ be an i-v $Q$-fuzzy set, then $\left\langle\langle\bar{A}\rangle_{L}\right\rangle_{R}=\langle\bar{A}\rangle=\left\langle\langle\bar{A}\rangle_{R}\right\rangle_{L}$. 
IV Q-Fuzzy Ideals Generated by an IV Q-Fuzzy Subset in Ordered Semi-groups

Proof: By Theorem 3.11, $\left\langle\langle\bar{A}\rangle_{L}\right\rangle_{R}$ is an i-v Q-fuzzy right ideal of $S$.

Clearly, $\left\langle\langle\bar{A}\rangle_{L}\right\rangle_{R}=\left[\left\langle\left\langle A^{-}\right\rangle_{L}\right\rangle_{R},\left\langle\left\langle A^{+}\right\rangle_{L}\right\rangle_{R}\right]$. For all $x, y \in S, q \in Q$

$\left\langle\left\langle A^{-}\right\rangle_{L}\right\rangle_{R}(x y, q)=\sup _{x y \leq a_{1} a_{2}}\left\langle A^{-}\right\rangle_{L}\left(a_{1}, q\right)=\sup _{x y \leq a_{1} a_{2} a_{1} \leq z_{1} z_{2}} A^{-}\left(z_{2}, q\right)$ and

$\left\langle\left\langle A^{-}\right\rangle_{L}\right\rangle_{R}(x y, q)=\sup _{y \leq y_{1} y_{2}}\left\langle A^{-}\right\rangle_{L}\left(y_{1}, q\right)=\sup _{y \leq y_{1} y_{2} y_{1} \leq w_{1} w_{2}} A^{-}\left(w_{2}, q\right)$

Obviously, $\left\langle\left\langle A^{-}\right\rangle_{L}\right\rangle_{R}(x y, q) \geq\left\langle\left\langle A^{-}\right\rangle_{L}\right\rangle_{R}(y, q)$.

Similarly we have $\left\langle\left\langle A^{+}\right\rangle_{L}\right\rangle_{R}(x y, q) \geq\left\langle\left\langle A^{+}\right\rangle_{L}\right\rangle_{R}(y, q)$.

It follows that,

$$
\begin{aligned}
\left\langle\langle\bar{A}\rangle_{L}\right\rangle_{R}(x y, q) & =\left[\left\langle\left\langle A^{-}\right\rangle_{L}\right\rangle_{R}(x y, q),\left\langle\left\langle A^{+}\right\rangle_{L}\right\rangle_{R}(x y, q)\right] \\
& \geq\left[\left\langle\left\langle A^{-}\right\rangle_{L}\right\rangle_{R}(y, q),\left\langle\left\langle A^{+}\right\rangle_{L}\right\rangle_{R}(y, q)\right]=\left\langle\langle\bar{A}\rangle_{L}\right\rangle_{R}(y, q)
\end{aligned}
$$

Hence $\left\langle\left\langle A^{-}\right\rangle_{L}\right\rangle$ is an i-v Q-fuzzy left ideal of $S$.

So $\left\langle\left\langle A^{-}\right\rangle_{L}\right\rangle_{R}$ is an i-v Q-fuzzy ideal of $S$.

Since $\bar{A} \subseteq\langle\bar{A}\rangle_{L} \subseteq\left\langle\langle\bar{A}\rangle_{L}\right\rangle_{R}$, we have $\left\langle\langle\bar{A}\rangle_{L}\right\rangle_{R} \supseteq \bar{A}$.

Suppose $\bar{B}$ is any i-v Q-fuzzy ideal of S such that $\bar{B} \supseteq \bar{A}$.

Since $\langle\bar{A}\rangle_{L}$ is the smallest i-v Q-fuzzy left ideal of $S$ containing $\bar{A}$,

we have $\bar{B} \supseteq\langle\bar{A}\rangle_{L}$. Also $\bar{B} \supseteq\left\langle\left\langle A^{-}\right\rangle_{L}\right\rangle_{R}$,

since $\left\langle\langle\bar{A}\rangle_{L}\right\rangle_{R}$ is the smallest i-v Q-fuzzy left ideal of $S$ containing $\langle\bar{A}\rangle_{L}$.

This show that $\left\langle\langle\bar{A}\rangle_{L}\right\rangle_{R}$ is a smallest i-v Q-fuzzy left ideal of $S$ containing $\bar{A}$.

Therefore $\left\langle\langle\bar{A}\rangle_{L}\right\rangle_{R}=\langle\bar{A}\rangle$.

Similarly we can prove that $\left\langle\langle\bar{A}\rangle_{R}\right\rangle_{L}=\langle\bar{A}\rangle$.

Hence $\left\langle\langle\bar{A}\rangle_{L}\right\rangle_{R}=\langle\bar{A}\rangle=\left\langle\langle\bar{A}\rangle_{R}\right\rangle_{L}$.

Definition 3.14. Let $\bar{A}$ be an $i$-v $Q$-fuzzy set. Then the smallest $i$-v $Q$-fuzzy interior ideal of $S$ containing $\bar{A}$ is called an $\boldsymbol{i}$-v $Q$-fuzzy interior ideal of $S$ generated by $\bar{A}$, denoted by $\langle\bar{A}\rangle_{I}$.

Theorem 3.15. Let $\bar{A}$ be an $i-v$-fuzzy set, then $\langle\bar{A}\rangle_{I}=\bar{J}$, where

$$
\bar{J}=\left[J^{-}, J^{+}\right] \text {such that } J^{-}(x, q)=\sup _{\substack{x \leq x_{1} x_{2} x_{3} \\ x_{1}, x_{2}, x_{3} \in S}} A^{-}\left(x_{2}, q\right)
$$

$J^{+}(x, q)=\sup _{\substack{x \leq x_{1} x_{1} x_{3} \\ x_{1}, x_{2}, x_{3} \in S}} A^{+}\left(x_{2}, q\right)$ for all $x \in S, q \in Q$. 
P.Murugadas, A.Arikrishnan and M.R.Thirumagal

Proof: For all $a \in S$ and $q \in Q$, we have

$J^{-}(a, q)=\sup _{a \leq x_{1} x_{2} x_{3}} A^{-}\left(x_{2}, q\right) \geq A^{-}(a, q)$ because $a \leq 1 a 1$.

Similarly $J^{+}(a, q) \geq A^{+}(a, q)$.

Therefore $\bar{J}(a, q)=\left[J^{-}(a, q), J^{+}(a, q)\right] \geq\left[A^{-}(a, q), A^{+}(a, q)\right]=\bar{A}(a, q)$.

Let $x, y \in S$, and $q \in Q$ such that $(x, q) \leq(y, q)$.

If $(y, q) \leq\left(x_{1} x_{2} x_{3}, q\right)$ then $(x, q) \leq\left(x_{1} x_{2} x_{3}, q\right)$.

Hence $J^{-}(y, q)=\sup _{y \leq x_{1} x_{2} x_{3}} A^{-}\left(x_{2}, q\right) \leq \sup _{x \leq x_{4} x_{5} x_{6}} A^{-}\left(x_{5}, q\right)=J^{-}(x, q)$.

Similarly $J^{+}(y, q) \leq J^{+}(x, q)$.

Hence $\bar{J}(x, q)=\left[J^{-}(x, q), J^{+}(x, q)\right] \geq\left[J^{-}(y, q), J^{+}(y, q)\right]=\bar{J}(y, q)$.

Also for all $x, y, z \in S$ and $q \in Q$,

if $(y, q) \leq\left(a_{1} a_{2} a_{3}, q\right)$ then $(x y z, q) \leq\left(\left(x a_{1}\right) a_{2}\left(a_{3} z\right), q\right)$.

Hence $J^{-}(y, q)=\sup _{y \leq a_{1} a_{2} a_{3}} A^{-}\left(a_{2}\right) \leq \sup _{x y z \leq b_{1} b_{2} b_{3}} A^{-}\left(b_{2}, q\right)=J^{-}(x y z, q)$.

Similarly $J^{+}(y, q) \geq J^{+}(x y z, q)$.

Thus $\bar{J}(y, q)=\left[J^{-}(y, q), J^{+}(y, q)\right] \leq\left[J^{-}(x y z, q), J^{+}(x y z, q)\right]=\bar{J}(x y z, q)$.

This shows that $\bar{J}$ is an i-v Q-fuzzy interior ideal of $S$ such that $\bar{B} \supseteq \bar{A}$.

Then for all $a \in S$ and $q \in Q$,

$$
\begin{aligned}
J^{-}(a, q) & =\sup _{a \leq a_{1} a_{2} a_{3}} A^{-}\left(a_{2}, q\right) \leq \sup _{a \leq a_{1} a_{2} a_{3}} B^{-}\left(a_{2}, q\right) \\
& \leq \sup _{a \leq a_{1} a_{2} a_{3}} B^{-}\left(a_{1} a_{2} a_{3}, q\right) \leq B^{-}(a, q) .
\end{aligned}
$$

Similarly $J^{+}(a, q) \leq B^{+}(a, q)$.

Hence $\bar{J}(a, q) \leq \bar{B}(a, q)$.

This shows that $\bar{J}$ is the smallest i-v Q-fuzzy interior ideal of $S$ containing $\bar{A}$, that is $\langle\bar{A}\rangle_{I}=\bar{J}$.

Theorem 3.16. Let $\bar{A}$ be an $i$-v $Q$-fuzzy set. An interval-valued $Q$-fuzzy subset $\bar{J}$ is an $i$-v $Q$-fuzzy interior ideal of $S$ generated by $\bar{A}$ iff $J^{-}$and $J^{+}$are fuzzy interior ideals of $S$ generated by $A^{-}$and $A^{+}$respectively.

Proof: The proof is similar to the proof of Theorem 3.12.

Definition 3.17. An $i-v Q-f u z z y$ sub semi-group $\bar{A}$ of $S$ is called an $i-v Q-f u z z y$ submonoid of $S$ if $\bar{A}(1, q) \geq \bar{A}(x, q)$ for all $x \in S$ and $q \in Q$.

Theorem 3.18. Let $\bar{A}$ be an $i-v$-fuzzy submonoid of $S$ then $\langle\bar{A}\rangle_{B}=\bar{J}$ where 
IV Q-Fuzzy Ideals Generated by an IV Q-Fuzzy Subset in Ordered Semi-groups

$$
\begin{aligned}
& J^{-}(x, q)=\sup _{x \leq x_{1} x_{2} x_{3}} \min \left\{A^{-}\left(x_{1}, q\right), A^{-}\left(x_{3}, q\right)\right\} \text { and } \\
& J^{+}(x, q)=\sup _{x \leq x_{1} x_{2} x_{3}} \min \left\{A^{+}\left(x_{1}, q\right), A^{+}\left(x_{3}, q\right)\right\} \text { for all } x \in S \text { and } q \in Q .
\end{aligned}
$$

Proof: For all $x \in S$ and $q \in Q$

$$
\begin{aligned}
J^{-}(x, q) & =\sup _{x \leq x_{1} x_{2} x_{3}} \min \left\{A^{-}\left(x_{1}, q\right), A^{-}\left(x_{3}, q\right)\right\} \\
& \geq \min \left\{A^{-}(1), A^{-}(x, q)\right\}=A^{-}(x, q)
\end{aligned}
$$

because $x \leq 1.1 . x$ and $\bar{A}(1, q) \geq \bar{A}(x, q)$ for all $x \in S$.

Similarly, we have $J^{+}(x, q) \geq A^{+}(x, q)$.

Therefore $\bar{J}(x, q) \geq \bar{A}(x, q)$ and so $\bar{J} \supseteq \bar{A}$.

Let $x, y \in S$ such that $x \leq y$.

If $y \leq x_{1} x_{2} x_{3}$ then $x \leq x_{1} x_{2} x_{3}$. Hence

$$
\begin{aligned}
J^{-}(y, q) & =\sup _{y \leq x_{1} x_{2} x_{3}} \min \left\{A^{-}\left(x_{1}, q\right), A^{-}\left(x_{3}, q\right)\right\} \\
& \leq \sup _{x \leq a_{1} a_{2} a_{3}} \min \left\{A^{-}\left(a_{1}, q\right), A^{-}\left(a_{3}, q\right)\right\}=J^{-}(x, q) .
\end{aligned}
$$

Similarly, we have $J^{+}(x, q) \geq J^{+}(y, q)$.

Thus $\bar{J}(x, q) \geq \bar{J}(y, q)$.

Also for all $x, y, z \in S$.

If $(x, q) \leq\left(x_{1} x_{2} x_{3}, q\right)$ and $(z, q) \leq\left(z_{1} z_{2} z_{3}, q\right)$,

then $(x y z, q) \leq\left(\left(x_{1} x_{2} x_{3}\right) y\left(z_{1} z_{2} z_{3}\right), q\right)$.

Hence

$$
\begin{aligned}
J^{-}(x y z, q) & =\sup _{x y z \leq a_{1} a_{2} a_{3}} \min \left\{A^{-}\left(a_{1}, q\right), A^{-}\left(a_{3}, q\right)\right\} \\
& \geq \sup _{\substack{\left.x y z \leq x_{1} \\
x \leq x_{1} x_{2} x_{z} z_{1} z_{2}\right) \\
x \leq x_{1} x_{2} x_{3}, z_{3} \leq z_{1} z_{2} z_{3}}} \min \left\{A^{-}\left(x_{1}, q\right), A^{-}\left(z_{3}, q\right)\right\} .
\end{aligned}
$$

We can write $A^{-}\left(x_{1}, q\right) \geq \min \left\{A^{-}\left(x_{1}\right), A^{-}\left(x_{3}, q\right)\right\}$,

$A^{-}\left(z_{3}, q\right) \geq \min \left\{A^{-}\left(z_{1}, q\right), A^{-}\left(z_{3}, q\right)\right\}$.

It follows that

$$
\begin{aligned}
J^{-}(x y z, q) & \geq \sup _{\substack{x y z \leq x_{1}\left(x_{2} x_{3}, z_{z} z_{2}\right) z_{3} \\
x \leq x_{1} x_{2} x_{3}, z z_{1} z_{1} z_{3}}} \min \left\{\min \left\{A^{-}\left(x_{1}, q\right), A^{-}\left(x_{3}, q\right)\right\}, \min \left\{A^{-}\left(z_{1}, q\right), A^{-}\left(z_{3}, q\right)\right\}\right\} \\
& =\min \left\{\sup _{x \leq x_{1} x_{2} x_{3}} \min \left\{A^{-}\left(x_{1}\right), A^{-}\left(x_{3}\right)\right\}, \sup _{z \leq z_{1} z_{2} z_{3}} \min \left\{A^{-}\left(z_{1}, q\right), A^{-}\left(z_{3}, q\right)\right\}\right\} \\
& =\min \left\{J^{-}(x, q), J^{-}(z, q)\right\} .
\end{aligned}
$$


P.Murugadas, A.Arikrishnan and M.R.Thirumagal

Similarly we have $J^{+}(x y z, q) \geq \min \left\{J^{+}(x, q), J^{+}(z, q)\right\}$.

Therefore

$$
\begin{aligned}
\bar{J}(x y z, q) & =\left[J^{-}(x y z, q), J^{+}(x y z, q)\right] \\
& \geq\left[\min \left\{J^{-}(x, q), J^{-}(z, q)\right\}, \min \left\{J^{+}(x, q), J^{+}(z, q)\right\}\right] \\
& =\operatorname{Min}^{i}\{\bar{J}(x, q), \bar{J}(z, q)\}
\end{aligned}
$$

and so, $\bar{J}(x y z, q) \geq \operatorname{Min}^{i}\{\bar{J}(x, q), \bar{J}(z, q)\}$.

Taking $\mathrm{y}=1$, we have $\bar{J}(x z, q) \geq \operatorname{Min}^{i}\{\bar{J}(x, q), \bar{J}(z, q)\}$.

This shows that $\bar{J}$ is an i-v Q-fuzzy bi-ideal of $S$.

Let $\bar{B}$ be an i-v Q-fuzzy bi-ideal of $S$ such that $\bar{B} \supseteq \bar{A}$.

Then for all $a \in S$, we have

$$
\begin{aligned}
J^{-}(a, q) & =\sup _{a \leq a_{1} a_{2} a_{3}} \min \left\{A^{-}\left(a_{1}, q\right), A^{-}\left(a_{3}, q\right)\right\} \\
& \leq \sup _{a \leq a_{1} a_{2} a_{3}} \min \left\{B^{-}\left(a_{1}, q\right), B^{-}\left(a_{3}, q\right)\right\} \leq \sup _{a \leq a_{1} a_{2} a_{3}} B^{-}\left(a_{1} a_{2} a_{3}, q\right) \leq B^{-}(a, q) .
\end{aligned}
$$

Similarly, we have $J^{+}(a, q) \leq B^{+}(a, q)$. Thus $\bar{J} \subseteq \bar{B}$.

Hence $\bar{J}$ is a smallest i-v Q-fuzzy bi-ideal of $S$ containing $\bar{A}$.

That is $\langle\bar{A}\rangle_{B}=\bar{J}$.

Theorem 3.19. Let $\bar{A}$ be an $i$-v $Q$-fuzzy submonoid of $S$. Then an $i$-v $Q$-fuzzy subset $\bar{J}$ is an $i$-v $Q$-fuzzy bi-ideal of $S$ generated by $\bar{A}$ if and only if $J^{-}$and $J^{+}$are $Q$-fuzzy bi-ideals of $S$ generated by $A^{-}$and $A^{+}$respectively.

Proof: The proof is similar to the proof of Theorem 3.18.

Theorem 3.20. Let $S$ be regular ordered semi-group and $\bar{A}$ be an $i-v Q$-fuzzy set, then $\langle\bar{A}\rangle_{B}=\bar{J}$ where

$$
\begin{aligned}
& J^{-}(x, q)=\sup _{x \leq x_{1} x_{2} x_{3}} \min \left\{A^{-}\left(x_{1}, q\right), A^{-}\left(x_{3}, q\right)\right\} \text { and } \\
& J^{+}(x, q)=\sup _{x \leq x_{1} x_{2} x_{3}} \min \left\{A^{+}\left(x_{1}, q\right), A^{+}\left(x_{3}, q\right)\right\} \text { for all } x \in S, q \in Q .
\end{aligned}
$$

Proof: From the proof of Theorem 3.18, it enough to prove that $\bar{J} \supseteq \bar{A}$.

For all $x \in S$ and $q \in Q$, we have 
IV Q-Fuzzy Ideals Generated by an IV Q-Fuzzy Subset in Ordered Semi-groups

$$
\begin{aligned}
J^{-}(x, q) & =\sup _{x \leq x_{1} x_{2} x_{3}} \min \left\{A^{-}\left(x_{1}, q\right), A^{-}\left(x_{3}, q\right)\right\} \\
& \geq \sup _{x \leq x a x} \min \left\{A^{-}(x, q), A^{-}(x, q)\right\} \\
& =A^{-}(x, q)
\end{aligned}
$$

Similarly, we have $J^{+}(x, q) \geq A^{+}(x, q)$.

Therefore, $\bar{J}(x, q) \geq \bar{A}(x, q)$ and so $\bar{J} \supseteq \bar{A}$.

Remark 3.21. Theorem 3.19 is also true for an $i-v$-fuzzy subset $\bar{A}$ of a regular ordered semi-group $S$.

\section{Conclusion}

Interval-valued Q-fuzzy ideals generated by an interval-valued Q-fuzzy subset on ordered semi-groups has been studied and some characterization are establisted.

\section{REFERENCES}

1. Y.B.Jun, J.Meng and X.L.Xin, On ordered filters of implicative semi-groups, Semi-group Forum, 54 (1997) 75-82.

2. N.Kehayopulu, On prime, weakly prime ideals in ordered semi-groups, Semi-group Forum, 44(3) (1992) 341-346.

3. N.Kehayopulu and M.Tsingelis, On intra-regular ordered semi-groups, Semi-group Forum, 46 (1993) 271-278.

4. N.Kehayopulu and M.Tsingelis, A note on fuzzy sets in semi-groups, Sci. Math., 5(3) (1999) 411-413.

5. N.Kehayopulu and M.Tsingelis, Fuzzy sets in ordered groupoids. Semi-group Forum, 65(1) (2002) 128-132.

6. N.Kehayopulu and M.Tsingelis, The embedding of an ordered groupid into a poe-groupid in terms of fuzzy sets, Information Sciences, 152 (2003) 231-236.

7. N.Kehayopulu and M.Tsingelis, Fuzzy bi-ideals in ordered semi-groups, Information Sciences, 171 (2005) 13-28.

8. N.Kehayopulu and M.Tsingelis, Regular ordered semi-groups in terms of fuzzy sets, Information Sciences, 171 (2005) 13-28.

9. N.Kehayopulu and M.Tsingelis, Fuzzy interior ideals in ordered semi-groups, Lovachevskli Journal of Mathematics, 21 (2006) 65-71.

10. N.Kuroki, On fuzzy semi-group, Information Sciences, 53(3) ( 1991) 203-236.

11. M.Pal and H.Rashmanlou, Irregular Interval-valued Fuzzy graphs, Annals of pure and Applied Mathematics, 1(2) (2012) 186-191.

12. R.Nagarajan and K.Balamurugan, On interval-valued bi-cubic vague subgroups, Annals of pure and Applied Mathematics, 6(2) (2014) 125-132.

13. A.L.Narayanan and T.Manikantan, Interval-valued fuzzy ideals generated by an interval-valued fuzzy subset in semi-groups, J. App. Maths and Computing, 20 (2006) 455-465.

14. P.Rajarajeswari and P.Dhanalakshmi, Interval-valued fuzzy soft matrix theory, Annals of pure and Applied Mathematics, 7(2) (2014) 61-72. 
P.Murugadas, A.Arikrishnan and M.R.Thirumagal

15. A.Rosenfeld, Fuzzy groups, Journal of Mathematical Analysis and Applications, 35(1971) 51-517

16. M.Shabir and Israr Ali Khan, Interval-valued fuzzy ideals generated by an interval-valued fuzzy subset in ordered semi-groups, Mathware and Soft Computing, 15 (2008) 263-272.

17. N.Thillaigovindan and V.Chinnadurai, Interval-valued fuzzy generalized bi-ideals, Proceedings of the National Conference on Algebra, Graph theory and their Applications, Department of Mathematics, Manonmaniam Sundaranar University, Narosa, (2009) 85-98.

18. L.A.Zadeh, Fuzzy Sets, Inform. and Control, 8(3)(1965) 338-353. 